\title{
SHIMURA CORRESPONDENCE FOR FINITE GROUPS
}

\author{
GORDAN SAVIN
}

\begin{abstract}
Let $\mathbb{Q}_{2}$ se the unique unramifed extension of the two-adic field $\mathbb{Q}_{2}$ of the degree $s$. Let $R$ be the ring of integers in $\mathbb{Q}_{2}$ set $G$ be a simply connected Chevalley group corresponding to an irreducible simply laced root system. Then the finite group $G(R / 4 R)$ has a two-fold central extension $G^{\prime}(R / 4 R)$ constructed by means of the Hilbert symbol on $\mathbb{Q}_{2}$. In this paper, we construct a natural correspondence between genuine representations of $G^{\prime}(R / 4 R)$ and representations of the Chevalley group $G(R / 2 R)$.
\end{abstract}

\section{Introduction}

Let $\Phi$ be an irreducible simply laced root system and let $G=G_{\mathrm{sc}}$ be the simply connected Chevalley group corresponding to $\Phi$. Let $F$ be a $p$-adic field and $R$ its ring of integers. Then $G(F)$ has a unique non-trivial central extension $G^{\prime}(F)$ by $\mu_{2}=\{ \pm 1\}$. If $p$ is odd, then the central extension splits (uniquely) over $G(R)$. In particular, $G(R)$ can be viewed as a subgroup of $G^{\prime}(F)$. On the other hand, if $F=\mathbb{Q}_{2^{s}}$ then for every $n>1$ the Hilbert symbol $(u, v)_{2}$ defines a nontrivial central extension $G^{\prime}\left(R / 2^{n} R\right)$ of $G\left(R / 2^{n} R\right)$ and the inverse image of $G(R)$ is a projective limit of $G^{\prime}\left(R / 2^{n} R\right)$, for $n>1$. Thus we are led to study genuine representations of $G^{\prime}(R / 4 R)$ in order to understand the simplest types of genuine representations of $G^{\prime}(F)$.

We now describe our results in more details. The kernel of the natural projection from $G(R / 4 R)$ to $G(R / 2 R)$ can be identified with $\mathfrak{g}(R / 2 R)$, the Lie algebra of $G$ over the residual field $R / 2 R$. Let $\mathfrak{g}^{\prime}(R / 2 R)$ be the preimage of $\mathfrak{g}(R / 2 R)$ in $G^{\prime}(R / 4 R)$. The group commutator of any two elements in $\mathfrak{g}^{\prime}(R / 2 R)$ is an element in $\mu_{2}$ and it depends only on the projection of the two elements onto $\mathfrak{g}(R / 2 R)$. Thus, the commutator defines a bilinear $\mu_{2}$-valued form $\omega(x, y)$ on $\mathfrak{g}(R / 2 R)$. Our first result is the description of this form. Let $\kappa$ be the Killing form on $\mathfrak{g}_{\mathbb{Z}}$, a Chevalley lattice in $\mathfrak{g}$. Then for all $x=X \otimes u$ and $y=Y \otimes v$ in $\mathfrak{g}_{\mathbb{Z}} \otimes(R / 2 R)=\mathfrak{g}(R / 2 R)$,

$$
\omega(x, y)=(1+2 u, 1+2 v)_{2}^{\kappa(X, Y)} .
$$

Let $Z$ be the kernel of the form $\omega$. Then $Z^{\prime}$, the inverse image of $Z$ in $\mathfrak{g}^{\prime}(R / 2 R)$, is the center of $\mathfrak{g}^{\prime}(R / 2 R)$. Let $\chi$ be a genuine character of $Z^{\prime}$. It is well known that there exists a unique irreducible representation $\rho_{\chi}$ of $\mathfrak{g}^{\prime}(R / 2 R)$ with the central character $\chi$. Our second result is that the representation $\rho_{\chi}$ extends to a representation of $G^{\prime}(R / 4 R)$, denoted by $\rho_{\chi}^{\prime}$. This extension is unique unless $G^{\prime}(R / 4 R)=\mathrm{SL}^{\prime}(\mathbb{Z} / 4 \mathbb{Z})$. Now the classification of genuine representations of $G^{\prime}(R / 4 R)$ is easy. Indeed, since $Z^{\prime}$ is contained in the center of $G^{\prime}(R / 4 R)$ any irreducible representation $\pi$ of $G^{\prime}(R / 4 R)$,

Received by the editors May 17, 2011.

1991 Mathematics Subject Classification. 20C33, 22E50. 
when restricted to $\mathfrak{g}^{\prime}(R / 2 R)$, is a multiple of $\rho_{\chi}$ for some character $\chi$ of $Z^{\prime}$. Thus one can canonically write

$$
\pi=\operatorname{Hom}_{\mathfrak{g}^{\prime}(R / 2 R)}\left(\rho_{\chi}^{\prime}, \pi\right) \otimes \rho_{\chi}^{\prime},
$$

where $G^{\prime}(R / 4 R)$ acts on $T \in \operatorname{Hom}_{\mathfrak{g}^{\prime}(R / 2 R)}\left(\rho_{\chi}^{\prime}, \pi\right)$ by $\pi(g) \circ T \circ \rho_{\chi}^{\prime}\left(g^{-1}\right)$. Since $T$ intertwines the action of $\mathfrak{g}^{\prime}(R / 2 R)$, this action descends to $G(R / 2 R)$. In this way, we have constructed a correspondence (in fact a functor) between representations of $G^{\prime}(R / 4 R)$ on which $Z^{\prime}$ acts by the genuine character $\chi$ and representations of $G(R / 2 R)$. This correspondence gives a bijection between equivalence classes of irreducible representations.

\section{Finite Chevalley groups}

Let $(\alpha \mid \beta)$ denote the inner product on $\Phi$ normalized such that $(\alpha \mid \alpha)=2$ for long roots. Co-roots can be identified with $\alpha^{\vee}:=\frac{2 \alpha}{(\alpha \mid \alpha)}$. Since $\Phi$ is simply laced, $\alpha^{\vee}=\alpha$. In particular, we can identify the root and the co-root lattices.

The root system $\Phi$ defines a split, simple Lie algebra $\mathfrak{g}$ over $\mathbb{Z}$. More precisely, we have a Chevalley lattice

$$
\mathfrak{g}_{\mathbb{Z}}=X \oplus_{\alpha \in \Phi} \mathbb{Z} \cdot E_{\alpha}
$$

where $X$ is the co-root lattice. The co-roots, considered as elements in the Chevalley lattice, will be denoted by $H_{\alpha}$.

We can define an invariant (Killing) form on $\mathfrak{g}$ by

$$
\left\{\begin{array}{l}
\kappa\left(H_{\alpha}, H_{\beta}\right)=\left(\alpha^{\vee} \mid \beta^{\vee}\right), \\
\kappa\left(E_{\alpha}, E_{-\alpha}\right)=1,
\end{array}\right.
$$

and 0 for any other combinations of Chevalley generators as entries of $\kappa$. Let $\mathfrak{g}(\mathbb{Z} / 2 \mathbb{Z})$ denote the Lie algebra over the finite field $\mathbb{Z} / 2 \mathbb{Z}$. Note that $\mathfrak{g}(\mathbb{Z} / 2 \mathbb{Z})$ is simply obtained by reducing the Chevalley lattice modulo 2 . The Killing form $\kappa$ can now be viewed as an invariant form on $\mathfrak{g}(\mathbb{Z} / 2 \mathbb{Z})$ with values in $\mathbb{Z} / 2 \mathbb{Z}$. Note that the kernel of $\kappa$ is equal to the kernel of the restriction of $\kappa$ to $X / 2 X$. This kernel is trivial if and only if the determinant of the Cartan matrix of the root system is odd.

Let $G=G_{\mathrm{sc}}$ be the simply connected Chevalley group corresponding to the root system $\Phi$. By fixing the Chevalley lattice, we have also fixed a structure of $G$ as a group scheme over $\mathbb{Z}$. Recall that there is a maximal, split torus $T$ in $G$ preserving root spaces in $\mathfrak{g}$ under the adjoint action. If $A$ is a ring, then $T(A) \cong X \otimes_{\mathbb{Z}} A^{\times}$. We shall also need the adjoint group $G_{\text {ad }}$. Let $T_{\text {ad }}$ be the maximal split torus in $G_{\text {ad }}$. Then $T_{\text {ad }}(A) \cong Y \otimes_{\mathbb{Z}} A^{\times}$, where $Y$ is the co-character lattice of $T_{\text {ad }}$. In the simply laced case $Y$ is the dual lattice to $X$ with respect to the product $(\alpha \mid \beta)$.

We shall be mostly interested in the case $A=R / 4 R$, where $R$ is the ring of integers in $\mathbb{Q}_{2}$. Since $R / 4 R$ is a local ring the group $G(R / 4 R)$ is generated by one-parameter subgroups $U_{\alpha} \simeq R / 4 R$ for all $\alpha$ in $\Phi$ (see [1], Proposition 1.6). The choice of Chevalley basis fixes an isomorphism of $R / 4 R$ and $U_{\alpha}, u \mapsto e_{\alpha}(u)$ for every $\alpha \in \Phi$. For example, if $G=\mathrm{SL}_{2}$ then $e_{\alpha}(u)$ and $e_{-\alpha}(u)$ are

$$
\left(\begin{array}{ll}
1 & u \\
0 & 1
\end{array}\right) \text { and }\left(\begin{array}{ll}
1 & 0 \\
u & 1
\end{array}\right) .
$$


For every $v$ in $(R / 4 R)^{\times}$define elements

$$
\left\{\begin{array}{l}
w_{\alpha}(v)=e_{\alpha}(v) e_{-\alpha}\left(-v^{-1}\right) e_{\alpha}(v) \\
h_{\alpha}(v)=w_{\alpha}(v) w_{\alpha}(-1)
\end{array}\right.
$$

If $G=\mathrm{SL}_{2}$ then $w_{\alpha}(v)$ and $h_{\alpha}(v)$ are

$$
\left(\begin{array}{cc}
0 & v \\
-v^{-1} & 0
\end{array}\right) \text { and }\left(\begin{array}{cc}
v & 0 \\
0 & v^{-1}
\end{array}\right)
$$

If $\Phi \neq A_{1}$, by a result of Stein ([8], Corollary 2.14), the group $G(R / 4 R)$ is abstractly generated by the one-parameter groups $U_{\alpha}$ modulo the relations

$$
\left[e_{\alpha}(u), e_{\beta}(v)\right]= \begin{cases}e_{\alpha+\beta}( \pm u v), & \text { if } \alpha+\beta \text { is a root, } \\ 1, & \text { if not, and }-\alpha \neq \beta\end{cases}
$$

and

$$
h_{\alpha}(u) h_{\alpha}(v)=h_{\alpha}(u v) .
$$

The group $G(R / 4 R)$ has a two-step filtration with $G(R / 2 R)$ as a quotient and a subgroup isomorphic to $\mathfrak{g}(R / 2 R)=\mathfrak{g}_{\mathbb{Z}} \otimes R / 2 R$. This isomorphism is explicitly given by

$$
\left\{\begin{array}{c}
h_{\alpha}(1+2 u) \mapsto H_{\alpha} \otimes u \\
e_{\alpha}(2 u) \mapsto E_{\alpha} \otimes u
\end{array}\right.
$$

Note that the relation (2.1) implies that the groups $G(R / 4 R)$ and $G(R / 2 R)$ are perfect if $\Phi \neq A_{1}$. The relation $\left[h_{\alpha}(v), e_{\alpha}(u)\right]=e_{\alpha}\left(\left(v^{2}-1\right) u\right)$ implies that $\mathrm{SL}_{2}(R / 4 R)$ and $\mathrm{SL}_{2}(R / 2 R)$ are also perfect if $|R / 2 R|>2$.

\section{Central extensions}

Assume that $\Phi \neq A_{1}$. Since the group $G(R / 4 R)$ is perfect, it has a universal central extension. The universal central extension (with some low rank exceptions) is given by the Steinberg group $G^{\prime \prime}(R / 4 R)$. The group $G^{\prime \prime}(R / 4 R)$ is generated by elements $e_{\alpha}^{\prime \prime}(u)$, for all $u \in R / 4 R$ and $\alpha \in \Phi$, satisfying $e_{\alpha}^{\prime \prime}(u) e_{\alpha}^{\prime \prime}(v)=e_{\alpha}^{\prime \prime}(u+v)$ and the relation (2.1). Define $h_{\alpha}^{\prime \prime}(v)$ in $G^{\prime \prime}(R / 4 R)$ in the same way as $h_{\alpha}(v)$ was defined in $G(R / 4 R)$. Then $h_{\alpha}^{\prime \prime}(v)$ do not necessarily satisfy the relation (2.2). Thus the Steinberg symbol $(u, v)_{S}$ is defined as the obstruction to the relation $(2.2)$ :

$$
(u, v)_{S}=h_{\alpha}^{\prime \prime}(u) h_{\alpha}^{\prime \prime}(v) h_{\alpha}^{\prime \prime}(u v)^{-1} .
$$

The symbol does not depend on the choice of the root $\alpha$. It is a central element in $G^{\prime \prime}(R / 4 R)$. The elements $(1+2 v, 1+2 u)_{S}$ are of order at most 2 and generate the kernel of the projection of $G^{\prime \prime}(R / 4 R)$ onto $G(R / 4 R)$ ([8], Theorem 3.10).

Let $(u, v)_{2}$ be the Hilbert symbol on $\mathbb{Q}_{2^{s}}$. When restricted to $(1+2 R) \times(1+2 R)$, the kernel of the Hilbert symbol is $1+4 R$ and, by passing to the quotient $1+2 R / 1+$ $4 R \cong R / 2 R$, the symbol induces a non-degenerate bilinear form on $R / 2 R$. The group $G(R / 4 R)$ has a non-trivial central extension by $\mu_{2}=\{ \pm 1\}$, denoted by $G^{\prime}(R / 4 R)$ obtained by specializing the Steinberg symbol to the Hilbert symbol:

$$
(u, v)_{S} \mapsto(u, v)_{2} .
$$


Let $e_{\alpha}^{\prime}(u)$ and $h_{\alpha}^{\prime}(v)$ in $G^{\prime}(R / 4 R)$ be the projections of $e_{\alpha}^{\prime \prime}(u)$ and $h_{\alpha}^{\prime \prime}(v)$ in $G^{\prime \prime}(R / 4 R)$, respectively. The elements $e_{\alpha}^{\prime}(u)$ satisfy the relation (2.1). However, the relation (2.2) is replaced by

$$
h_{\alpha}^{\prime}(u) h_{\alpha}^{\prime}(v)=h_{\alpha}^{\prime}(u v) \cdot(u, v)_{2} .
$$

The elements $h_{\alpha}^{\prime}(u)$ and $h_{\beta}^{\prime}(v)$ generally do not commute. Their commutator is

$$
\left[h_{\alpha}^{\prime}(u), h_{\beta}^{\prime}(v)\right]=(u, v)_{2}^{\left(\alpha^{\vee} \mid \beta^{\vee}\right)} .
$$

Define $\mathrm{SL}_{2}^{\prime}(R / 4 R)$ as a subgroup of $G^{\prime}(R / 4 R)$ generated by elements $e_{\alpha}^{\prime}(u)$ and $e_{-\alpha}^{\prime}(u)$, for all $u \in R / 4 R$ and $\alpha$ one fixed root. This definition does not depend on the choice of the root system $\Phi \neq A_{1}$ and the root $\alpha$ in $\Phi$. In this way, we have defined $G^{\prime}(R / 4 R)$ for all simply laced root systems including $A_{1}$.

The group $G^{\prime}(R / 4 R)$ is perfect unless $G^{\prime}(R / 4 R)=\mathrm{SL}_{2}^{\prime}(\mathbb{Z} / 4 \mathbb{Z})$, for the same reason as $G(R / 4 R)$. The conjugation action of $G^{\prime}(R / 4 R)$ on $G^{\prime}(R / 4 R)$ descends down to an action of $G(R / 4 R)$ on $G^{\prime}(R / 4 R)$. In fact, an element $t=\lambda \otimes v$ in $T(R / 4 R)=$ $X \otimes(\mathbb{Z} / 4 \mathbb{Z})^{\times}$acts on the generating elements of $G^{\prime}(R / 4 R)$ by

$$
t e_{\alpha}^{\prime}(u) t^{-1}=e_{\alpha}^{\prime}\left(v^{(\lambda \mid \alpha)} u\right)
$$

Moreover, since the formula (3.1) makes sense for any $t=\lambda \otimes s$ in $T_{\text {ad }}(4)=Y \otimes$ $(\mathbb{Z} / 4 \mathbb{Z})^{\times}$, the adjoint group $G_{\mathrm{ad}}(R / 4 R)$ acts on $G^{\prime}(R / 4 R)$.

We have the following diagram of groups:

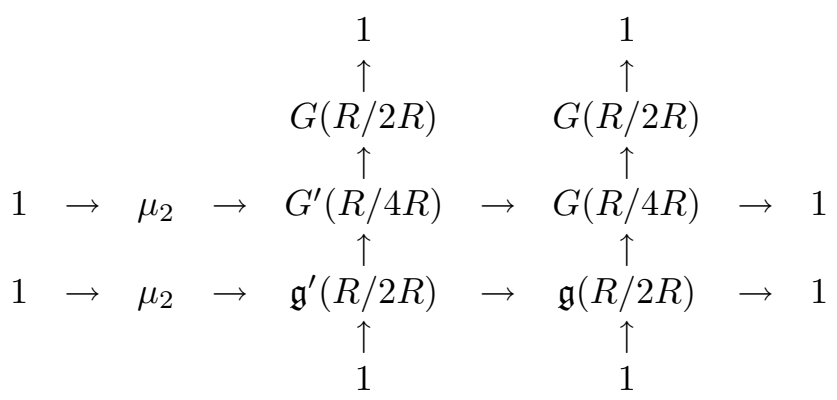

We now describe the central extension $\mathfrak{g}^{\prime}(R / 2 R)$ of $\mathfrak{g}(R / 2 R)$ appearing in the diagram. (See [3] and [6] for more on the subject of extensions of elementary twogroups.) We can define a symplectic form $\omega$ on $\mathfrak{g}(R / 2 R)$ with values in $\mu_{2}$ by

$$
\omega(x, y)=\left[x^{\prime}, y^{\prime}\right]
$$

where $x^{\prime}$ and $y^{\prime}$ are any two elements in $\mathfrak{g}^{\prime}(R / 2 R)$ that project to $x$ and $y$, respectively, and $\left[x^{\prime}, y^{\prime}\right]$ denotes the group commutator.

Proposition 3.1. Let $\kappa$ be the Killing form on $\mathfrak{g}_{\mathbb{Z}}$. Then, for any two elements $X \otimes u$ and $Y \otimes v$ in $\mathfrak{g}_{\mathbb{Z}} \otimes R / 2 R=\mathfrak{g}(R / 2 R)$,

$$
\omega(X \otimes u, Y \otimes v)=(1+2 u, 1+2 v)_{2}^{\kappa(X, Y)} .
$$

Proof. There are several cases to consider. Assume first that $x=H_{\alpha} \otimes u$ and $y=$ $H_{\beta} \otimes v$. We can take $x^{\prime}=h_{\alpha}^{\prime}(1+2 u)$ and $y^{\prime}=h_{\beta}^{\prime}(1+2 v)$. Since

$$
\left[h_{\alpha}^{\prime}(1+2 u), h_{\beta}^{\prime}(1+2 v)\right]=(1+2 u, 1+2 v)_{2}^{\left(\alpha^{\vee} \mid \beta^{\vee}\right)},
$$


this case has been checked. Next, assume that $x=E_{\alpha} \otimes u$ and $y=E_{\beta} \otimes v$ where $\alpha \neq-\beta$. Then $\kappa\left(E_{\alpha}, E_{\beta}\right)=0$. We can take $x^{\prime}=e_{\alpha}^{\prime}(2 u)$ and $y^{\prime}=e_{\beta}^{\prime}(2 v)$. Since

$$
\left[e_{\alpha}^{\prime}(2 u), e_{\beta}^{\prime}(2 v)\right]=e_{\alpha+\beta}^{\prime}( \pm 4 u v)=1
$$

in $G^{\prime}(R / 4 R)$, this case has been also checked. If $\beta=-\alpha$, then this is Corollary 2.9 in [8]. The remaining cases are trivial.

Let $Z$ be the kernel of the form $\omega$. In order to describe $Z$, it suffices to describe the kernel of the Killing form considered modulo 2. Recall that the kernel of the Killing form on $\mathfrak{g}(\mathbb{Z} / 2 \mathbb{Z})$ is equal to the kernel of the Killing form restricted on $X / 2 X$. Let $\hat{X}$ be a lattice, $X \subseteq \hat{X} \subseteq Y$, such that $\hat{X} / X$ is the two-torsion in $Y / X$. Since $Y$ is dual to $X$, it follows that $2 \hat{X} / 2 X$ is the kernel of the Killing form. It follows that

$$
Z \cong(2 \hat{X} / 2 X) \otimes(1+2 R / 1+4 R) \cong(2 \hat{X} / X) \otimes(R / 2 R) .
$$

Proposition 3.2. Let $Z^{\prime}$ be the center of the nilpotent group $\mathfrak{g}^{\prime}(R / 2 R)$.

(1) The group $Z^{\prime}$ is the preimage of $Z$, the kernel of $\omega$.

(2) The group $Z^{\prime}$ is contained in the center of $G^{\prime}(R / 4 R)$.

(3) The adjoint action of $T_{\mathrm{ad}}(R / 4 R)$ on $Z^{\prime}$ induces a transitive action on the set of genuine characters of $Z^{\prime}$.

Proof. The first statement is obvious. Let $t$ in $T^{\prime}(R / 4 R)$ such that its image in $T(R / 4 R)$ is $t=\lambda \otimes v$. Then the conjugation action of $t$ on a generating element $e_{\alpha}^{\prime}(u)$ is given by

$$
t e_{\alpha}^{\prime}(u) t^{-1}=e_{\alpha}^{\prime}\left(v^{(\lambda \mid \alpha)} u\right) .
$$

If $t$ is in $Z^{\prime}$ then $\lambda \in 2 \hat{X}$ and $v \in 1+2 R$. But then $v^{\left(\lambda \mid \alpha^{\vee}\right)}=1$ and $Z^{\prime}$ is in the center of $G^{\prime}(R / 4 R)$. This proves the second statement. Finally, the conjugation action of an element $t=\lambda \otimes v$ in $T_{\mathrm{ad}}(R / 4 R) \cong Y \otimes(R / 4 R)^{\times}$on $h_{\alpha}^{\prime}(u)$ is given by

$$
t h_{\alpha}^{\prime}(v) t^{-1}=h_{\alpha}^{\prime}(v) \cdot(v, u)_{2}^{\left(\lambda \mid \alpha^{\vee}\right)} .
$$

Since the lattice $Y$ is dual to $X$ this formula shows that the group $T_{\mathrm{ad}}(R / 4 R)$ acts transitively on the set of genuine characters of $Z^{\prime}$.

\section{Main results}

Fix a genuine character $\chi$ of $Z^{\prime}$, the center of $\mathfrak{g}^{\prime}(R / 2 R)$. Let $S \subseteq \mathfrak{g}(R / 2 R)$ be a maximal subspace such that the bilinear form $\omega$ is trivial on $S$. Let $S^{\prime} \subseteq \mathfrak{g}^{\prime}(R / 2 R)$ be the inverse image of $S$. Then $S^{\prime}$ is a maximal abelian subgroup of $\mathfrak{g}^{\prime}(R / 2 R)$ and $\chi$ extends (in more than one way) to a character of $S^{\prime}$. Let $\chi_{S}$ be one extension. Define

$$
\rho_{\chi}=\operatorname{Ind}_{S^{\prime}}^{\mathfrak{g}^{\prime}(R / 2 R)}\left(\chi_{S}\right) .
$$

It is not difficult to see that $\rho_{\chi}$ does not depend on the choice of $\chi_{S}$ and that it is the unique irreducible representation of $\mathfrak{g}^{\prime}(R / 2 R)$ with the central character $\chi$. Indeed, the restriction of $\rho_{\chi}$ to $S^{\prime}$ is the sum of all characters of $S^{\prime}$ extending $\chi$, thus the claim follows from the Frobenius reciprocity and Mackey's irreducibility criterion. We note that the square of the dimension of $\rho_{\chi}$ is

$$
\operatorname{dim}\left(\rho_{\chi}\right)^{2}=\frac{\left|\mathfrak{g}^{\prime}(R / 2 R)\right|}{\left|Z^{\prime}\right|}=\frac{|\mathfrak{g}(R / 2 R)|}{|Z|},
$$


where, we remind the reader, $Z$ is the kernel of the pairing $\omega$ on $\mathfrak{g}(R / 2 R)$. In the following table we give the size of $Z$ :

\begin{tabular}{|r||r|r|r|r|r|r|r|}
\hline$\Phi$ & $A_{2 n-1}$ & $A_{2 n}$ & $D_{2 n-1}$ & $D_{2 n}$ & $E_{6}$ & $E_{7}$ & $E_{8}$ \\
\hline \hline$|Z|$ & 1 & $2^{s}$ & $2^{s}$ & $2^{2 s}$ & 1 & $2^{s}$ & 1 \\
\hline
\end{tabular}

Proposition 4.1. The representation $\rho_{\chi}$ of $\mathfrak{g}^{\prime}(R / 2 R)$ extends to a representation of $G^{\prime}(R / 4 R)$. This extension is denoted by $\rho_{\chi}^{\prime}$. The extension is unique unless $G(R / 4 R)=$ $\mathrm{SL}_{2}(\mathbb{Z} / 4 \mathbb{Z})$.

Proof. We note that $G(R / 4 R)$ acts by conjugation on irreducible representations of $\mathfrak{g}^{\prime}(R / 2 R)$. Since the isomorphism class of $\rho_{\chi}$ depends on the central character $\chi$, and $Z^{\prime}$ is central in $G^{\prime}(R / 4 R)$, we see that the conjugation by $G(R / 4 R)$ does not change the isomorphism class of $\rho_{\chi}$. In particular, $\rho_{\chi}$ gives rise to a projective representation of $G(R / 4 R)$. By Theorem 2.13 in [8], the Steinberg group $G^{\prime \prime}(R / 4 R)$ is universal if the rank of $\Phi$ is at least 5 . Assume this. Then the projective representation lifts to a representation of $G^{\prime \prime}(R / 4 R)$. Let $\rho_{\chi}^{\prime}$ denote this representation. We claim that this representation descends to $G^{\prime}(R / 4 R)$. Indeed, for every $u \in R, e_{\alpha}^{\prime \prime}(2 u) \in G^{\prime \prime}(R / 4 R)$ and $e_{\alpha}^{\prime}(2 u) \in \mathfrak{g}^{\prime}(R / 2 R)$ are a scalar multiple of each other (when acting by $\rho_{\chi}^{\prime}$ and $\rho_{\chi}$ respectively). In particular, their commutators coincide. Since

$$
(1+2 u, 1+2 v)_{S}=\left[e_{\alpha}^{\prime \prime}(2 u), e_{-\alpha}^{\prime \prime}(2 v)\right]=\left[e_{\alpha}^{\prime}(2 u), e_{-\alpha}^{\prime}(2 v)\right]=(1+2 u, 1+2 v)_{2},
$$

we see that the Steinberg symbol acts through its Hilbert specialization, i.e., $G^{\prime \prime}(R / 4 R)$ acts through its quotient $G^{\prime}(R / 4 R)$. Note, however, that the restriction of $\rho_{\chi}^{\prime}$ to $\mathfrak{g}^{\prime}(R / 2 R)$ is not necessarily isomorphic to $\rho_{\chi}$. It may be isomorphic to a twist of $\rho_{\chi}$ by a character of $\mathfrak{g}(R / 2 R)$. Any such twist is isomorphic to $\rho_{\chi^{\prime}}$ for a (possibly) different genuine character $\chi^{\prime}$ of $Z^{\prime}$. Since the maximal torus $T_{\mathrm{ad}}(R / 4 R)$ of the adjoint group acts transitively on the set of all genuine characters of $Z^{\prime}$, we can conjugate by an element in $T_{\mathrm{ad}}(R / 4 R)$, if necessary, to construct an extension of $\rho_{\chi}$ to $G^{\prime}(R / 4 R)$. The uniqueness of extension is clear since $G^{\prime}(R / 4 R)$ is perfect unless $G^{\prime}(R / 4 R)=\mathrm{SL}_{2}^{\prime}(\mathbb{Z} / 4 \mathbb{Z})$.

To deal with low rank groups of type $A_{m}$ and $D_{m}$ we proceed as follows. Let $\Phi_{0} \subseteq \Phi$ be a root subsystem of the same type as $\Phi$ but of the rank $m-1$. Fix $\Phi^{+}$, a set of positive roots. Let $P=M U$ be a maximal parabolic subgroup of $G$ such that $U$ is generated by the root groups $U_{\alpha}$ for $\alpha \in \Phi^{+} \backslash \Phi_{0}$. Then $G_{0}=[M, M]$ is a simply connected Chevalley group corresponding to $\Phi_{0}$.

Let $Z_{0}^{\prime}$ be the center of $\mathfrak{g}_{0}^{\prime}(R / 2 R)$. If $m$ is odd then $Z^{\prime} \subseteq Z_{0}^{\prime}$ and every genuine character $\chi$ of $Z^{\prime}$ is the restriction of $2^{s}$ genuine characters $\chi_{1}, \ldots, \chi_{2^{s}}$ of $Z_{0}^{\prime}$. Let $U_{2}$ be the subgroup of $G^{\prime}(R / 4 R)$ generated $e_{\alpha}^{\prime}(2 u)$ for $\alpha \in \Phi^{+} \backslash \Phi_{0}$. Let $\rho_{\chi}^{U_{2}}$ be the subspace of $U_{2}$-fixed vectors in $\rho_{\chi}$. Then, as representations of $\mathfrak{g}_{0}^{\prime}(R / 2 R)$,

$$
\rho_{\chi}^{U_{2}} \cong \rho_{\chi_{1}} \oplus \cdots \oplus \rho_{\chi_{2} s}
$$

Thus, if $\rho_{\chi}$ extends to a representation of $G^{\prime}(R / 4 R)$ then, by taking $U_{2}$-fixed vectors, $G_{0}^{\prime}(R / 4 R)$ acts naturally on $\rho_{\chi_{1}}, \ldots, \rho_{\chi_{2} s}$. Similarly, if $m$ is even then $Z_{0}^{\prime} \subseteq Z^{\prime}$ and every genuine character $\chi$ of $Z^{\prime}$ is the restriction of $2^{s}$ genuine characters $\chi_{1}, \ldots, \chi_{2^{s}}$ 
of $Z_{0}^{\prime}$. Then, as representations of $\mathfrak{g}_{0}^{\prime}(R / 2 R)$,

$$
\rho_{\chi_{1}}^{U_{2}} \cong \cdots \cong \rho_{\chi_{2}}^{U_{2}} \cong \rho_{\chi}
$$

and $G_{0}^{\prime}(R / 4 R)$ acts naturally on $\rho_{\chi}$.

If $\sigma$ is a representation of $G(R / 2 R)$ then, after inflating $\sigma$ to $G^{\prime}(R / 4 R), \sigma \otimes \rho_{\chi}^{\prime}$ is a genuine representation of $G^{\prime}(R / 4 R)$. Clearly, $\sigma \otimes \rho_{\chi}^{\prime}$ is an irreducible representation of $G^{\prime}(R / 4 R)$ if and only if $\sigma$ is an irreducible representation of $G(R / 2 R)$.

Theorem 4.1. Let $\chi$ be a genuine character of $Z^{\prime}$, the center of $\mathfrak{g}^{\prime}(R / 2 R) \subseteq G^{\prime}(R / 4 R)$. The map $\sigma \mapsto \sigma \otimes \rho_{\chi}^{\prime}$ gives a one to one correspondence between isomorphism classes of irreducible representations of $G(R / 2 R)$ and irreducible representations of $G^{\prime}(R / 4 R)$ such that $Z^{\prime}$ acts by the character $\chi$.

Proof. Let $\pi$ be an irreducible representation of $G^{\prime}(R / 4 R)$ such that $Z^{\prime}$ acts by the character $\chi$. Clearly, the restriction of $\pi$ to $\mathfrak{g}^{\prime}(R / 2 R)$ is a multiple of $\rho_{\chi}$. Let

$$
\sigma=\operatorname{Hom}_{\mathfrak{g}^{\prime}(R / 2 R)}\left(\rho_{\chi}^{\prime}, \pi\right) .
$$

Note that $\sigma$ is naturally a $G^{\prime}(R / 4 R)$-module with the action of $g$ in $G^{\prime}(R / 4 R)$ given by

$$
\sigma(g)(T)=\pi(g) \circ T \circ \rho_{\chi}^{\prime}\left(g^{-1}\right),
$$

for every $T$ in $\sigma$. Since $T$ intertwines the action of $\mathfrak{g}^{\prime}(R / 2 R), \sigma$ descends to a representation of $G(R / 2 R)$. Moreover, the natural map $T \otimes w \mapsto T(w)$ gives an isomorphism of $\sigma \otimes \rho_{\chi}^{\prime}$ and $\pi$. The theorem is proved.

We finish this paper with a remark on the relevance of our results to the local Shimura correspondence for two-adic groups. Let $Z_{2}$ be the algebraic subgroup of $G$ defined as the two-torsion of the center of $G$. Let $\hat{G}$ be the algebraic group defined as the quotient of $G$ by $Z_{2}$. The co-character lattice of $\hat{G}$ is $\hat{X}$. We remind the reader that $X \subseteq \hat{X} \subseteq Y$ and $\hat{X} / X$ is the two-torsion in $Y / X$. In particular, if $\hat{T}$ is a maximal torus of $\hat{\hat{G}}$ then $\hat{T}(F) \cong \hat{X} \otimes F^{\times}$. One expects that there is a Shimura correspondence between genuine representations of $G^{\prime}\left(\mathbb{Q}_{2^{s}}\right)$ and representations of the linear group $\hat{G}\left(\mathbb{Q}_{2^{s}}\right)$, by analogy with real groups [2] and $p$-adic groups with $p$ odd [7]. Under this correspondence, once we have fixed the genuine character $\chi$ of $Z^{\prime}$, irreducible generic representations of $G^{\prime}\left(\mathbb{Q}_{2^{s}}\right)$ containing the type $\rho_{\chi}^{\prime}$ should correspond to irreducible unramifed representations of $\hat{G}\left(\mathbb{Q}_{2}\right)$. More generally, irreducible genuine representations of $G^{\prime}\left(\mathbb{Q}_{2^{s}}\right)$ containing the type $\rho_{\chi^{\prime}}^{\prime}$ (now $\chi^{\prime}$ is any genuine character of $Z^{\prime}$ ) should correspond to irreducible unramifed representations of $\hat{G}\left(\mathbb{Q}_{2}\right)$ containing a one-dimensional type of conductor 4 depending on $\chi^{\prime}$. More precisely, let $\hat{G}(R)^{\text {der }}$ be the derived subgroup of $\hat{G}(R)$. Then

$$
\hat{G}(R) / \hat{G}(R)^{\operatorname{der}} \cong(\hat{X} / X) \otimes R^{\times} .
$$

Since $\hat{X} / X$ is two-torsion, any character $\mu$ of $\hat{G}(R)$ is necessarily quadratic. Since $(R / 2 R)^{\times}$is odd, $\mu$ is determined by its restriction on $(\hat{X} / X) \otimes(1+2 R)$. We say that 
$\mu$ is of conductor 4 if it is trivial on $(\hat{X} / X) \otimes(1+4 R)$. Thus quadratic characters of $\hat{G}(R)$ of conductor 4 correspond to characters of

$$
(\hat{X} / X) \otimes(1+2 R / 1+4 R) \cong(\hat{X} / X) \otimes(R / 2 R) \cong Z .
$$

Thus, irreducible genuine representations of $G^{\prime}\left(\mathbb{Q}_{2^{s}}\right)$ containing the type $\rho_{\chi^{\prime}}^{\prime}$ for should correspond to irreducible unramifed representations of $\hat{G}\left(\mathbb{Q}_{2}\right)$ containing a one-dimensional type $\mu$ where, abusing the notation, $\chi^{\prime}=\chi \cdot \mu$.

\section{Acknowledgments}

This paper has been motivated by the case $G=\mathrm{SL}_{2}$ and $F=\mathbb{Q}_{2}$ discussed in a joint work with Loke [5]. The work of Gurevich and Hadani [4] provides a version of Proposition 4.1 for the root system $C_{n}$. I would like to thank Zeev Rudnick for pointing out this reference to me. Thanks are also due to Dick Gross for an enlightening discussion on central extensions of elementary 2-groups, and to the referee. This work has been supported by an NSF grant DMS-0852429.

\section{References}

[1] E. Abe, Chevalley groups over local rings, Tôhoku Math. J. 21 (1969), 474-494.

[2] J. Adam, D. Barbasch, A. Paul, P. Trapa and D. Vogan, Unitary Shimura correspondences for split real groups, J. Amer. Math. Soc. 20 (2007), 701-751.

[3] R. Greiss, Automorphisms of extra-special groups and non-vanishing degree 2 cohomology, Pacific J. Math. 48 (1973), 403-422.

[4] S. Gurevich and R. Hadani, The Weil representation in characteristic two, Adv. Math. 230 (2012), 894-926.

[5] H. Y. Loke and G. Savin, Representations of the two-fold central extension of $\mathrm{SL}_{2}\left(\mathbb{Q}_{2}\right), \mathrm{Pacific}$ J. Math. 247(2) (2010), 435-454.

[6] D. Quillen, The mod 2 cohomology rings of extra-special 2-groups and the spinor groups, Math. Ann. 194 (1977), 197-212.

[7] G. Savin, On unramified representations of covering groups, J. Reine Angew. Math. 566 (2004), 111-134.

[8] M. Stein, Surjective stability in dimension 0 for $K_{2}$ and related functors, Trans. Amer. Math. Soc. 178 (1973), 165-191.

Department of Mathematics, University of Utah, Salt Lake City, UT 84112, USA

E-mail address: savin@math.utah.edu 